QUARTERLY OF APPLIED MATHEMATICS

VOLUME LXVI, NUMBER 3

SEPTEMBER 2008, PAGES 423-435

S 0033-569X(08)01118-7

Article electronically published on June 6, 2008

\title{
OVERLAPPING DOMAIN PROBLEMS IN THE CRACK THEORY WITH POSSIBLE CONTACT BETWEEN CRACK FACES
}

\author{
BY \\ ALEXANDER KHLUDNEV (Lavrentyev Institute of Hydrodynamics of the Russian Academy of \\ Sciences, Novosibirsk 630090, Russia) \\ AND \\ ATUSI TANI (Department of Mathematics, 3-14-1 Keio University, Yokohama 223-8522, Japan)
}

\begin{abstract}
The paper is concerned with the analysis of a new class of overlapping domain problems for elastic bodies having cracks. Inequality type boundary conditions are imposed on the crack faces. We prove an existence of invariant integrals and analyze the asymptotic behavior of the solution. It is shown that the limit problem describes an equilibrium state for the elastic body with a thin inclusion.
\end{abstract}

1. Introduction. It is well known that the linear crack theory is characterized by linear boundary conditions on crack faces [1, 2, 3]. Usually linear models lead to inconsistency with applications since, in the frames of such models, opposite crack faces may penetrate each other. In recent years the crack theory with possible contact between crack faces has been advanced very much (see 4]). In particular, solvability of boundary value problems was established for different constitutive laws, shape sensitivity problems were analyzed including differentiability of energy functionals with respect to a crack length, new approaches (such as the smooth domain method and the fictitious domain method) for investigation of similar problems were elaborated, and so on. Nonlinear boundary conditions considered in this theory describe a mutual nonpenetration between crack faces. Consequently, from the standpoint of applications, this theory is more suitable.

In this paper, an overlapping domain problem in the crack theory with possible contact between crack faces is considered, which allows us to analyze a new class of models useful in applications. A different approach we employ to the problem is that we can, in fact, consider a Riemann surface having two sheets with glue and nonpenetration boundary conditions on the crack faces.

Received July 1, 2006.

2000 Mathematics Subject Classification. Primary 49J40, 49J10, 35J65, 35J70.

E-mail address: khlud@hydro.nsc.ru

E-mail address: tani@math.keio.ac.jp

(C) 2008 Brown University Reverts to public domain 28 years from publication 
A number of crack problems require overlapping domain approaches for their mathematical modeling in applications.

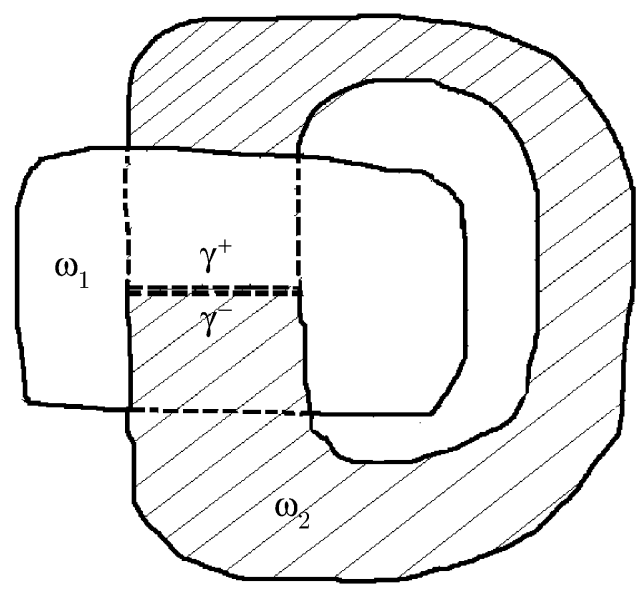

FIG. 1

To consider an example, we may imagine two flat sheets of ice, which we label $\omega_{1}$ and $\omega_{2}$; see Fig. 1. The sheet $\omega_{1}$ has a cut (crack) $\gamma$ with crack faces $\gamma^{ \pm}$. Assuming that the ice sheets are elastic, we are looking for in-plane displacement fields $u=\left(u_{1}, u_{2}\right)$ in $\omega_{1}$ and $v=\left(v_{1}, v_{2}\right)$ in $\omega_{2}$. In applications it is interesting to impose glue conditions between displacement fields $u, v$ defined on $\gamma^{+}, \gamma^{-}$, respectively. Simultaneously, nonpenetration conditions on $\gamma^{+}, \gamma^{-}$for fields $v, u$, respectively, are imposed. To simplify the geometry of the problem, in the paper we consider coinciding domains $\omega_{1}, \omega_{2}$ with both glue conditions and inequality type conditions on $\gamma^{ \pm}$describing a mutual nonpenetration between the crack faces.

2. Problem formulation. Let $\Omega \subset R^{2}$ be a bounded domain with Lipschitz boundary $\Gamma$, and let $\gamma \subset \Omega$ be a smooth curve (crack) without selfintersections. Denote $\Omega_{\gamma}=\Omega \backslash \bar{\gamma}$

Assume that $\gamma$ can be extended up to a closed curve $\Sigma$ of the class $C^{1,1}$ belonging to $\Omega$. In this case $\Omega$ is divided into two subdomains, $\Omega_{1}$ and $\Omega_{2}$. The boundary of $\Omega_{1}$ coincides with $\Sigma$, and the boundary of $\Omega_{2}$ is $\Sigma \cup \Gamma$; see Fig 2. It is supposed that $\gamma$ does not contain its tip points and can be extended in such a way that $\Omega$ is divided into two subdomains $\omega_{1}, \omega_{2}$ with Lipschitz boundaries $\partial \omega_{1}, \partial \omega_{2}$, and meas $\left(\partial \omega_{i} \cap \Gamma\right)>0, i=1,2$. Denote by $\nu=\left(\nu_{1}, \nu_{2}\right)$ a unit normal vector to $\gamma$ and to $\Sigma$, directed to the domain $\Omega_{2}$.

We first provide a variational formulation of the problem. Introduce the Sobolev space

$$
H_{\Gamma}^{1}\left(\Omega_{\gamma}\right)=\left\{w \in H^{1}\left(\Omega_{\gamma}\right) \mid w=0 \text { on } \Gamma\right\}
$$

and a set of admissible displacements

$$
\begin{gathered}
K=\left\{(u, v) \in\left(H_{\Gamma}^{1}\left(\Omega_{\gamma}\right)\right)^{2} \times\left(H_{\Gamma}^{1}\left(\Omega_{\gamma}\right)\right)^{2} \mid\right. \\
\left.u^{+}=v^{-}, u^{-} \nu \leq v^{+} \nu \quad \text { a.e. on } \gamma\right\} .
\end{gathered}
$$




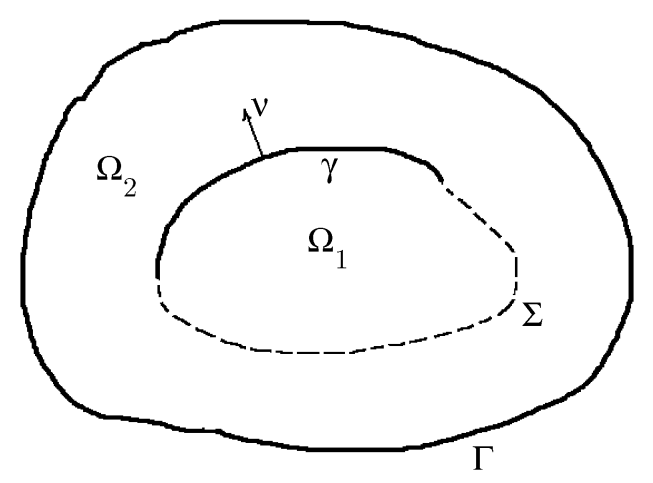

FIG. 2

Here $u^{ \pm}, v^{ \pm}$fit to the positive and negative crack faces $\gamma^{ \pm}$with respect to the normal vector $\nu ; u=\left(u_{1}, u_{2}\right), v=\left(v_{1}, v_{2}\right)$.

Consider the functional

$$
\Pi(u, v)=\frac{1}{2} \int_{\Omega_{\gamma}}\{p(u) \varepsilon(u)+P(v) \varepsilon(v)\}-\int_{\Omega_{\gamma}}\{g u+h v\}
$$

and the minimization problem

$$
\inf _{(u, v) \in K} \Pi(u, v) .
$$

Here $\varepsilon(u)=\left\{\varepsilon_{i j}(u)\right\}, \varepsilon(v)=\left\{\varepsilon_{i j}(v)\right\}$ are strain tensor components, $\varepsilon_{i j}(u)=\frac{1}{2}\left(u_{i, j}+\right.$ $\left.u_{j, i}\right), u_{i, j}=\frac{\partial u_{i}}{\partial x_{j}}, i, j=1,2,\left(x_{1}, x_{2}\right) \in \Omega_{\gamma} ; p(u)=\left\{p_{i j}(u)\right\}, P(v)=\left\{P_{i j}(v)\right\}, i, j=1,2$, are stress tensor components,

$$
p(u)=A \varepsilon(u), P(v)=B \varepsilon(v),
$$

where $A=\left\{a_{i j k l}\right\}, B=\left\{b_{i j k l}\right\}, i, j, k, l=1,2$, are given elasticity tensors with the usual properties of symmetry and positive definiteness,

$$
a_{i j k l}=a_{j i k l}=a_{k l i j}, a_{i j k l} \xi_{k l} \xi_{i j} \geq c|\xi|^{2}, \quad c>0, \forall \xi_{i j}, \xi_{i j}=\xi_{j i} .
$$

For simplicity we assume that $A, B$ are constant tensors. All functions with two lower indices are assumed to be symmetric in those indices, i.e. $p_{i j}(u)=p_{j i}(u)$, etc. The functions $g=\left(g_{1}, g_{2}\right) \in L^{2}\left(\Omega_{\gamma}\right), h=\left(h_{1}, h_{2}\right) \in L^{2}\left(\Omega_{\gamma}\right)$ are given.

The problem (11) has a unique solution satisfying the variational inequality

$$
\begin{gathered}
(u, v) \in K, \\
\int_{\Omega_{\gamma}}\{p(u) \varepsilon(\bar{u}-u)+P(v) \varepsilon(\bar{v}-v)\} \\
-\int_{\Omega_{\gamma}}\{g(\bar{u}-u)+h(\bar{v}-v)\} \geq 0 \quad \forall(\bar{u}, \bar{v}) \in K .
\end{gathered}
$$

Below we provide a differential formulation of the problem (2), (3). In particular, a complete system of boundary conditions on $\gamma$ will be determined. 
Substitute in (3) test functions of the form $(\bar{u}, \bar{v})=(u, v) \pm(\varphi, \psi),(\varphi, \psi) \in C_{0}^{\infty}\left(\Omega_{\gamma}\right), \varphi=$ $\left(\varphi_{1}, \varphi_{2}\right), \psi=\left(\psi_{1}, \psi_{2}\right)$. It provides the equations

$$
\begin{aligned}
-\operatorname{div} p(u) & =g \text { in } \Omega_{\gamma}, \\
-\operatorname{div} P(v) & =h \text { in } \Omega_{\gamma},
\end{aligned}
$$

holding in the sense of distributions.

Substitute next in (3) test functions of the form $(\bar{u}, \bar{v})=(u, v)+(\varphi, \psi),(\varphi, \psi) \in K$. This implies the inequality

$$
\int_{\Omega_{\gamma}}\{p(u) \varepsilon(\varphi)+P(v) \varepsilon(\psi)\}-\int_{\Omega_{\gamma}}\{g \varphi+h \psi\} \geq 0 .
$$

We can change the integration domain $\Omega_{\gamma}$ by $\Omega_{1} \cup \Omega_{2}$ here and integrate by parts which, by (4), (5), yields the relation

$$
\begin{gathered}
-\left\langle p\left(u^{+}\right) \nu, \varphi^{+}\right\rangle_{1 / 2, \Sigma}+\left\langle p\left(u^{-}\right) \nu, \varphi^{-}\right\rangle_{1 / 2, \Sigma} \\
-\left\langle P\left(v^{+}\right) \nu, \psi^{+}\right\rangle_{1 / 2, \Sigma}+\left\langle P\left(v^{-}\right) \nu, \psi^{-}\right\rangle_{1 / 2, \Sigma} \geq 0 \quad \forall(\varphi, \psi) \in K .
\end{gathered}
$$

Here $\langle\cdot, \cdot\rangle_{1 / 2, \Sigma}$ means a duality pairing between $H^{1 / 2}(\Sigma)$ and its dual $H^{-1 / 2}(\Sigma)$. In deriving (77) we use Green's formula for the domains $\Omega_{1}, \Omega_{2}$ with boundaries $\partial \Omega_{1}, \partial \Omega_{2}$ (see 4])

$$
-\int_{\Omega_{i}} w \cdot \operatorname{div} \sigma=\int_{\Omega_{i}} \varepsilon(w) \cdot \sigma-\langle\sigma n, w\rangle_{1 / 2, \partial \Omega_{i}}, \quad i=1,2,
$$

valid for all $\sigma=\left\{\sigma_{i j}\right\}, i, j=1,2 ; \sigma, \operatorname{div} \sigma \in L^{2}\left(\Omega_{i}\right), w=\left(w_{1}, w_{2}\right) \in H^{1}\left(\Omega_{i}\right)$, where $n=\left(n_{1}, n_{2}\right)$ is a unit external normal vector to $\partial \Omega_{i}, i=1,2$, and $\sigma n=\left(\sigma_{1 j} n_{j}, \sigma_{2 j} n_{j}\right)$.

Notice that if $\varphi=\psi=0$ on $\gamma$, the inequality (7) can be written in the form of equality

$$
\langle[p(u) \nu], \varphi\rangle_{1 / 2, \Sigma \backslash \bar{\gamma}}^{00}+\langle[P(v) \nu], \psi\rangle_{1 / 2, \Sigma \backslash \bar{\gamma}}^{00}=0,
$$

where $[q]=q^{+}-q^{-}$is a jump of $q$ through $\gamma$, and $\langle\cdot, \cdot\rangle_{1 / 2, \Sigma \backslash \bar{\gamma}}^{00}$ is a duality pairing between $H_{00}^{-1 / 2}(\Sigma \backslash \bar{\gamma})$ and $H_{00}^{1 / 2}(\Sigma \backslash \bar{\gamma})$. The norm in the space $H_{00}^{1 / 2}(\Sigma \backslash \bar{\gamma})$ is introduced by the following formula:

$$
\|w\|_{1 / 2, \Sigma \backslash \bar{\gamma}}^{00}=\left(\|w\|_{1 / 2, \Sigma \backslash \bar{\gamma}}^{2}+\int_{\Sigma \backslash \bar{\gamma}} \frac{w^{2}}{\rho}\right)^{1 / 2},
$$

where $\|w\|_{1 / 2, \Sigma \backslash \bar{\gamma}}$ is the norm in the space $H^{1 / 2}(\Sigma \backslash \bar{\gamma})$ and $\rho(x)=\operatorname{dist}(x, \partial(\Sigma \backslash \bar{\gamma}))$. Consequently, taking into account an arbitrariness of $\varphi, \psi$ in (8), it follows that

$$
[p(u) \nu]=0,[P(v) \nu]=0 \text { in the sense of } H_{00}^{-1 / 2}(\Sigma \backslash \bar{\gamma}) .
$$

Now take $\varphi=\psi \in C_{0}^{\infty}(\Omega)$ in (7), $\varphi=\left(\varphi_{1}, \varphi_{2}\right), \psi=\left(\psi_{1}, \psi_{2}\right)$, which gives

$$
[p(u) \nu+P(v) \nu]=0 \text { in the sense of } H^{-1 / 2}(\Sigma) .
$$


It is possible to take in (3) test functions of the form $(\bar{u}, \bar{v})=(0,0),(\bar{u}, \bar{v})=2(u, v)$ and sum the relations obtained. This provides the equality

$$
\int_{\Omega_{\gamma}}\{p(u) \varepsilon(u)+P(v) \varepsilon(v)\}-\int_{\Omega_{\gamma}}\{g u+h v\}=0 .
$$

By (4), (5), the integration by parts can be performed here. Hence we derive

$$
\begin{gathered}
-\left\langle p\left(u^{+}\right) \nu, u^{+}\right\rangle_{1 / 2, \Sigma}+\left\langle p\left(u^{-}\right) \nu, u^{-}\right\rangle_{1 / 2, \Sigma} \\
-\left\langle P\left(v^{+}\right) \nu, v^{+}\right\rangle_{1 / 2, \Sigma}+\left\langle P\left(v^{-}\right) \nu, v^{-}\right\rangle_{1 / 2, \Sigma}=0 .
\end{gathered}
$$

This relation means that the left-hand side of inequality (7) is equal to zero provided that $(\varphi, \psi)=(u, v)$.

Notice that the above relations (7)-(11) hold for any closed curve $\Sigma$ extending $\gamma$ and satisfying the required smoothness $C^{1,1}$.

Consider next a neighborhood $V_{x_{0}}$ of a point $x_{0} \in \gamma$. We take $(\varphi, \psi) \in K$ such that $\operatorname{supp} \varphi \subset \bar{V}_{x_{0}}^{+}, \operatorname{supp} \psi \subset \bar{V}_{x_{0}}^{-}, \varphi^{+}=\psi^{-}$on $\gamma$. Here $V_{x_{0}}^{+}=V_{x_{0}} \cap \Omega_{2}, V_{x_{0}}^{-}=V_{x_{0}} \cap \Omega_{1}$. Substitution of $(\varphi, \psi)$ into (7) implies that

$$
-\left\langle p\left(u^{+}\right) \nu, \varphi^{+}\right\rangle_{1 / 2, \Sigma}+\left\langle P\left(v^{-}\right) \nu, \psi^{-}\right\rangle_{1 / 2, \Sigma}=0 .
$$

Consequently,

$$
\left\langle p\left(u^{+}\right) \nu-P\left(v^{-}\right) \nu, \varphi\right\rangle_{1 / 2, \gamma}^{00}=0 \quad \forall \varphi=\left(\varphi_{1}, \varphi_{2}\right) \in H_{00}^{1 / 2}(\gamma)
$$

which means

$$
p\left(u^{+}\right) \nu=P\left(v^{-}\right) \nu \text { in the sense of } H_{00}^{-1 / 2}(\gamma)
$$

Similarly, if supp $\varphi \subset \bar{V}_{x_{0}}^{-}$, supp $\psi \subset \bar{V}_{x_{0}}^{+}, \varphi^{-}=\psi^{+}$on $\gamma$, from (7) it follows that

$$
\left\langle p\left(u^{-}\right) \nu, \varphi^{-}\right\rangle_{1 / 2, \Sigma}-\left\langle P\left(v^{+}\right) \nu, \psi^{+}\right\rangle_{1 / 2, \Sigma}=0
$$

and hence

$$
p\left(u^{-}\right) \nu=P\left(v^{+}\right) \nu \text { in the sense of } H_{00}^{-1 / 2}(\gamma) .
$$

Now we can take a smooth function $\varphi=\left(\varphi_{1}, \varphi_{2}\right)$ such that $\operatorname{supp} \varphi \subset \bar{V}_{x_{0}}^{-}, \varphi^{-} \nu \leq 0$ on $\gamma$. In this case $(\varphi, 0) \in K$, and (7) implies

$$
\left\langle p\left(u^{-}\right) \nu, \varphi^{-}\right\rangle_{1 / 2, \Sigma} \geq 0 \quad \forall \varphi \in\left(H_{\Gamma}^{1}\left(\Omega_{\gamma}\right)\right)^{2}, \varphi^{-} \nu \leq 0 \text { on } \gamma, \operatorname{supp} \varphi \subset \bar{V}_{x_{0}}^{-} .
$$

Consequently

$$
\left\langle p\left(u^{-}\right) \nu, \varphi\right\rangle_{1 / 2, \gamma}^{00} \geq 0 \quad \forall \varphi=\left(\varphi_{1}, \varphi_{2}\right) \in H_{00}^{1 / 2}(\gamma), \varphi \nu \leq 0 \text { on } \gamma .
$$

Due to the representation

$$
p\left(u^{-}\right) \nu=p_{\nu}\left(u^{-}\right) \nu+p_{\tau}\left(u^{-}\right)
$$

with $p_{\nu}\left(u^{-}\right)=p_{i j}\left(u^{-}\right) \nu_{j} \nu_{i}, p_{\tau}\left(u^{-}\right)=\left(p_{\tau}^{1}\left(u^{-}\right), p_{\tau}^{2}\left(u^{-}\right)\right)$, from (14) it follows that

$$
\begin{aligned}
& p_{\nu}\left(u^{-}\right) \leq 0 \text { in the sense of } H_{00}^{-1 / 2}(\gamma), \\
& p_{\tau}\left(u^{-}\right)=0 \text { in the sense of } H_{00}^{-1 / 2}(\gamma) .
\end{aligned}
$$


By repeating the above arguments we can derive

$$
\begin{aligned}
& P_{\nu}\left(v^{+}\right) \leq 0 \text { in the sense of } H_{00}^{-1 / 2}(\gamma), \\
& P_{\tau}\left(v^{+}\right)=0 \text { in the sense of } H_{00}^{-1 / 2}(\gamma)
\end{aligned}
$$

The values $P_{\nu}\left(v^{+}\right), P_{\tau}\left(v^{+}\right)$are introduced similar to those of (15). In virtue of (13), we also obtain

$$
p_{\nu}\left(u^{-}\right)=P_{\nu}\left(v^{+}\right) \text {on } \gamma
$$

By (9), (12), (13), (17), (19), (20) and by the equality $u^{+}=v^{-}$on $\gamma$, the relation (11) formally can be written as $p_{\nu}\left(u^{-}\right)\left(u^{-} \nu-v^{+} \nu\right)=0$ on $\gamma$.

Therefore, now we are in a position to give a differential formulation of the problem (2), (3). Namely, it is necessary to find functions $u=\left(u_{1}, u_{2}\right), v=\left(v_{1}, v_{2}\right)$, such that

$$
\begin{array}{r}
-\operatorname{div} p(u)=g \text { in } \Omega_{\gamma}, \\
-\operatorname{div} P(v)=h \text { in } \Omega_{\gamma}, \\
p(u)=A \varepsilon(u), P(v)=B \varepsilon(v) \text { in } \Omega_{\gamma}, \\
u=v=0 \text { on } \Gamma, \\
u^{+}=v^{-}, u^{-} \nu \leq v^{+} \nu \text { on } \gamma, \\
p\left(u^{+}\right) \nu=P\left(v^{-}\right) \nu \text { on } \gamma, \\
p_{\nu}\left(u^{-}\right)=P_{\nu}\left(v^{+}\right) \leq 0, p_{\tau}\left(u^{-}\right)=P_{\tau}\left(v^{+}\right)=0 \text { on } \gamma, \\
p_{\nu}\left(u^{-}\right)\left(u^{-} \nu-v^{+} \nu\right)=0 \text { on } \gamma .
\end{array}
$$

Notice that if a solution of (21) $-(28)$ is quite smooth, then the variational inequality (2), (3) can be derived from (21)-(28). Hence, the system of boundary conditions (24)(28) is complete. We know that boundary conditions (26) -(28) are, in fact, fulfilled in the sense of (7), (11).

We should remark that (21)-(22) are equilibrium equations, and (23) represents the Hooke law. Relations (24) provide the bodies clamping on $\Gamma$. The first equality (25) and (26) describe the glue condition on $\gamma$. The inequality in (25) with (27), (28) describes the nonpenetration condition between the crack faces with zero friction.

It is interesting to notice a fulfillment of the equation

$$
-\operatorname{div}(p(u)+P(v))=g+h \text { in } \Omega
$$

in the sense of distributions. Remember that $\Omega$ is the domain with smooth boundary $\Gamma$. Indeed, consider the distribution $\operatorname{div}(p(u)+P(v))+g+h$ in $\Omega$. Let $\varphi=\left(\varphi_{1}, \varphi_{2}\right) \in C_{0}^{\infty}(\Omega)$ 
be any function. By (10), (21), (22), we have

$$
\begin{gathered}
(\operatorname{div}(p(u)+P(v))+g+h, \varphi)_{\Omega} \\
=-\int_{\Omega_{1}}(p(u)+P(v)) \varepsilon(\varphi)-\int_{\Omega_{2}}(p(u)+P(v)) \varepsilon(\varphi)+\int_{\Omega}(g+h) \varphi \\
=\int_{\Omega_{1}} \operatorname{div}(p(u)+P(v)) \varphi+\int_{\Omega_{2}} \operatorname{div}(p(u)+P(v)) \varphi+\int_{\Omega}(g+h) \varphi \\
+\langle[p(u) \nu+P(v) \nu], \varphi\rangle_{1 / 2, \Sigma}=0,
\end{gathered}
$$

which proves the assertion.

3. Differentiation of energy functionals. Invariant integrals. In this section we assume $\gamma=(0,1) \times\{0\} ; g=\left(g_{1}, g_{2}\right) \in C^{1}\left(R^{2}\right), h=\left(g_{1}, g_{2}\right) \in C^{1}\left(R^{2}\right)$. For convenience the domain $\Omega_{\gamma}$ will be denoted by $\Omega_{0}$. In what follows a perturbation of the geometrical domain $\Omega_{0}$ is considered. In the perturbed domain, a problem similar to that of (2), (3) is formulated. The perturbation is characterized by a parameter $\delta$. We differentiate the energy functional with respect to $\delta$ at the point $\delta=0$. It turns out that the derivative of the energy functional can be written as an integral over closed curves surrounding crack tips. Moreover, the integral does not depend on the chosen curves. The only requirement is the sufficient smoothness of the curves.

Now consider a transformation of the domain $\Omega_{0}$,

$$
y=\Phi_{\delta}(x), x \in \Omega_{0}, y \in \Omega_{\delta},
$$

where

$$
\Phi_{\delta}(x)=x+\delta F(x), F(x)=\left(F^{1}(x), 0\right),
$$

and $F^{1} \in W_{l o c}^{1, \infty}\left(R^{2}\right)$ is a given function. For small $\delta$ the transformation (29) establishes a one-to-one mapping between $\Omega_{0}$ and $\Omega_{\delta}$. Denote $\Gamma_{\delta}=\Phi_{\delta}(\Gamma), \gamma_{\delta}=\Phi_{\delta}(\gamma)$ and assume that for a small $\delta$ the normal $\nu$ to $\gamma$ is transformed into the normal vector $\nu$ to $\gamma_{\delta}$.

Let us formulate a family of problems perturbed with respect to (21)-(28) (or to (22) (32) ). We have to find functions $u_{\delta}=\left(u_{\delta 1}, u_{\delta 2}\right), v_{\delta}=\left(v_{\delta 1}, v_{\delta 2}\right)$, such that

$$
\begin{array}{r}
-\operatorname{div} p\left(u_{\delta}\right)=g \text { in } \Omega_{\delta}, \\
-\operatorname{div} P\left(v_{\delta}\right)=h \text { in } \Omega_{\delta}, \\
p\left(u_{\delta}\right)=A \varepsilon\left(u_{\delta}\right), P\left(v_{\delta}\right)=B \varepsilon\left(v_{\delta}\right) \text { in } \Omega_{\delta}, \\
u_{\delta}=v_{\delta}=0 \text { on } \Gamma_{\delta}, \\
u_{\delta}^{+}=v_{\delta}^{-}, u_{\delta}^{-} \nu \leq v_{\delta}^{+} \nu \text { on } \gamma_{\delta}, \\
p\left(u_{\delta}^{+}\right) \nu=P\left(v_{\delta}^{-}\right) \nu \text { on } \gamma_{\delta}, \\
p_{\nu}\left(u_{\delta}^{-}\right)=P_{\nu}\left(v_{\delta}^{+}\right) \leq 0, p_{\tau}\left(u_{\delta}^{-}\right)=P_{\tau}\left(v_{\delta}^{+}\right)=0 \text { on } \gamma_{\delta}, \\
p_{\nu}\left(u_{\delta}^{-}\right)\left(u_{\delta}^{-} \nu-v_{\delta}^{+} \nu\right)=0 \text { on } \gamma_{\delta} .
\end{array}
$$


The problem (30)-(37) for a small $\delta$ can be written in the variational form. Namely, denote

$$
\begin{gathered}
K^{\delta}=\left\{(u, v) \in\left(H_{\Gamma_{\delta}}^{1}\left(\Omega_{\delta}\right)\right)^{2} \times\left(H_{\Gamma_{\delta}}^{1}\left(\Omega_{\delta}\right)\right)^{2} \mid u^{+}=v^{-},\right. \\
\left.u^{-} \nu \leq v^{+} \nu \quad \text { a.e. on } \gamma_{\delta}\right\} .
\end{gathered}
$$

Then the problem (30)-(37) corresponds to the following variational inequality having a unique solution

$$
\begin{gathered}
\left(u_{\delta}, v_{\delta}\right) \in K^{\delta}, \\
\int_{\Omega_{\delta}}\left\{p\left(u_{\delta}\right) \varepsilon\left(\bar{u}-u_{\delta}\right)+P\left(v_{\delta}\right) \varepsilon\left(\bar{v}-v_{\delta}\right)\right\} \\
-\int_{\Omega_{\delta}}\left\{g\left(\bar{u}-u_{\delta}\right)+h\left(\bar{v}-v_{\delta}\right)\right\} \geq 0 \quad \forall(\bar{u}, \bar{v}) \in K^{\delta} .
\end{gathered}
$$

We see that the problem (38), (39) coincides with (21), (3) as $\delta=0$. To simplify the notations in the sequel, the solution $\left(u_{0}, v_{0}\right)$ will be denoted by $(u, v)$.

Now consider the energy functional for the problem (38), (39):

$$
E\left(u_{\delta}, v_{\delta} ; \Omega_{\delta}\right)=\frac{1}{2} \int_{\Omega_{\delta}}\left\{p\left(u_{\delta}\right) \varepsilon\left(u_{\delta}\right)+P\left(v_{\delta}\right) \varepsilon\left(v_{\delta}\right)\right\}-\int_{\Omega_{\delta}}\left\{g u_{\delta}+h v_{\delta}\right\} .
$$

Since the transformation (29) provides a one-to-one correspondence between $K$ and $K^{\delta}$, we can use a technique of differentiation of energy functionals developed in [5], 6] for finding the derivative

$$
I=\left.\frac{d}{d \delta} E\left(u_{\delta}, v_{\delta} ; \Omega_{\delta}\right)\right|_{\delta=0} .
$$

Moreover, the following formula is valid:

$$
\begin{aligned}
I=\int_{\Omega_{0}}\left\{\frac{1}{2} F_{, 1}^{1}(p(u) \varepsilon(u)+P(v) \varepsilon(v))\right. & \left.-\left(p_{i j}(u) u_{i, 1}+P_{i j}(v) v_{i, 1}\right) F_{, j}^{1}\right\} \\
& -\int_{\Omega_{0}}\left\{\left(F^{1} g_{i}\right)_{, 1} u_{i}+\left(F^{1} h_{i}\right)_{, 1} v_{i}\right\},
\end{aligned}
$$

where $(u, v)$ is the solution of the problem (38), (39) corresponding to $\delta=0$, i.e. of the problem (2), (3).

We should remark that the solution $u, v$ of the problem (2), (3) has an additional regularity up to points of $\gamma$. Namely, for any point $x_{0} \in \gamma$, there exists a neighborhood $V_{x_{0}}$ such that $u, v \in H^{2}\left(V_{x_{0}}^{ \pm}\right)$(see [4, page 100). This implies that we can perform an integration by parts in (40). For many concrete fields $F=\left(F^{1}, 0\right)$ this integration may lead to the so-called invariant integrals over closed curves. Consider an example. Let a support of a smooth function $\theta$ belong to a small neighborhood $U_{1}$ of the point $(1,0)$, and $\theta=1$ in a neighborhood $U_{2}$ of the point $(1,0), U_{2} \subset U_{1}$. Denote by $n=\left(n_{1}, n_{2}\right)$ a unit internal normal vector to the boundary $\partial U_{2}$. Assume that boundaries $\partial U_{i}, i=1,2$, satisfy the Lipschitz condition. Transformation (29) is chosen as follows:

$$
y_{1}=x_{1}+\delta \theta\left(x_{1}, x_{2}\right), y_{2}=x_{2},
$$


where $\left(x_{1}, x_{2}\right) \in \Omega_{0},\left(y_{1}, y_{2}\right) \in \Omega_{\delta}$. In this case $F(x)=(\theta(x), 0)$. The formula (40) can be rewritten in the following form:

$$
\begin{aligned}
I=\int_{\Omega_{0}}\left\{\frac{1}{2} \theta, 1(p(u) \varepsilon(u)+P(v) \varepsilon(v))-\right. & \left.\left(p_{i j}(u) u_{i, 1}+P_{i j}(v) v_{i, 1}\right) \theta_{, j}\right\} \\
& -\int_{\Omega_{0}}\left\{\left(\theta g_{i}\right)_{, 1} u_{i}+\left(\theta h_{i}\right)_{, 1} v_{i}\right\} .
\end{aligned}
$$

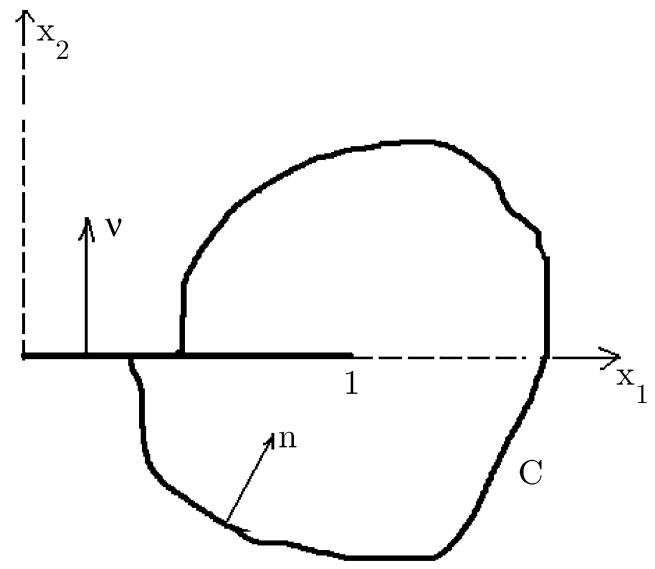

FIG. 3

Assume that $g=h=0$ in $U_{2}$ and perform an integration by parts in (41) by using (21), (22). This leads to the integration over the closed curve $\left(\partial U_{2}\right) \cap \bar{\Omega}_{0}$ :

$$
I=\int_{C}\left\{\frac{1}{2} n_{1}(p(u) \varepsilon(u)+P(v) \varepsilon(v))-\left(p_{i j}(u) u_{i, 1}+P_{i j}(v) v_{i, 1}\right) n_{j}\right\} .
$$

Here $\left(\partial U_{2}\right) \cap \bar{\Omega}_{0}$ is denoted by $C$; see Fig. 3 .

In deriving (42) from (41) we have used the relations

$$
\begin{aligned}
& p_{i 2}\left(u^{+}\right) u_{i, 1}^{+}=P_{i 2}\left(v^{-}\right) v_{i, 1}^{-} \text {on } \gamma, \\
& p_{i 2}\left(u^{-}\right) u_{i, 1}^{-}=P_{i 2}\left(v^{+}\right) v_{i, 1}^{+} \text {on } \gamma .
\end{aligned}
$$

It is clear that (43) follows from (26) and the first relation of (25). Let us prove (44). First note that the second relation of (25) has the form $u_{2}^{-}-v_{2}^{+} \leq 0$ on $\gamma$. By (28), we have

$$
p_{22}\left(u^{-}\right)\left(u_{2}^{-}-v_{2}^{+}\right)=0 \text { on } \gamma .
$$

As we remarked, the functions $u, v$ have $H^{2}$-regularity up to points of $\gamma$; hence $u, v$ are continuous up to points of $\gamma^{ \pm}$. On the set

$$
N=\left\{x \in \gamma \mid u_{2}^{-}(x)-v_{2}^{+}(x)<0\right\},
$$


in view of (45), we have $p_{22}\left(u^{-}\right)=0$, and hence

$$
p_{22}\left(u^{-}\right)\left(u_{2,1}^{-}-v_{2,1}^{+}\right)=0 .
$$

On the complement to $N$, i.e. on the set $\gamma \backslash N$, the equality $u_{2}^{-}-v_{2}^{+}=0$ holds, and consequently $u_{2,1}^{-}-v_{2,1}^{+}=0$ (see [7, Chapter 2, Th. A.1) which implies (46) on $\gamma \backslash N$. Therefore, the following equality takes place:

$$
p_{22}\left(u^{-}\right)\left(u_{2,1}^{-}-v_{2,1}^{+}\right)=0 \text { on } \gamma
$$

which, by the first relation of (27), is equivalent to

$$
p_{22}\left(u^{-}\right) u_{2,1}^{-}=P_{22}\left(v^{+}\right) v_{2,1}^{+} \text {on } \gamma \text {. }
$$

This completes the proof of (44) since, by (27), the relation (47) coincides with (44).

If a part of the curve $C$ coincides with any part of $\gamma$ (as is shown in Fig. 3), we can take the function values on the positive crack face $\gamma^{+}$or on the negative crack face $\gamma^{-}$ in the formula (42). To prove this statement it suffices to take into account (43), (44).

It is important that the integral (42) does not depend on $C$ since the integral is equal to the derivative $I$. In the crack theories similar integrals are called invariant integrals.

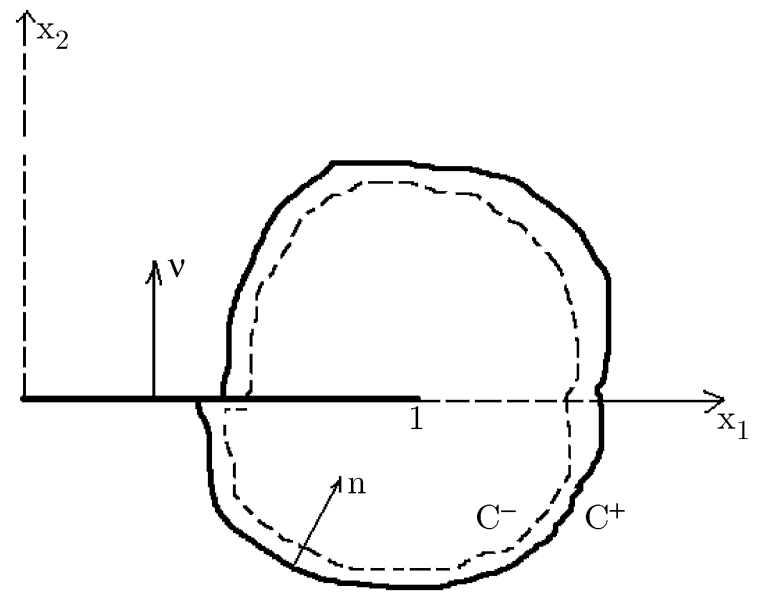

FIG. 4

We can interpret the problem (21)-(28) in terms of Riemann surfaces. The functions $u, v$ are determined in $\Omega_{\gamma}$ with boundary conditions (25)-(28) on $\gamma$. Hence we can consider the Riemann surface with two sheets. If the function $u$ is understood to be defined on the lower sheet, and, respectively, $v$ is defined on the upper sheet, the invariant integral 
(42) can be presented as a sum of two integrals:

$$
\begin{gathered}
I=I^{-}(u)+I^{+}(v), \\
I^{-}(u)=\int_{C^{-}}\left\{\frac{1}{2} n_{1} p(u) \varepsilon(u)-p_{i j}(u) u_{i, 1} n_{j}\right\}, \\
I^{+}(v)=\int_{C^{+}}\left\{\frac{1}{2} n_{1} P(v) \varepsilon(v)-P_{i j}(v) v_{i, 1} n_{j}\right\},
\end{gathered}
$$

where $C^{-}, C^{+}$belong to the lower and upper sheets, respectively. In such a case we must simultaneously choose the function values on the positive or negative crack faces $\gamma^{ \pm}$in (48), (49); see Fig. 4.

To conclude the section, note that linear crack problems were analyzed in $[8]$. The results related to linear perturbed crack problems can be found in [9], [10], [11].

4. Asymptotic analysis. Thin inclusions. From the standpoint of applications it is interesting to analyze passages to limits when parameters of the model (21)-(28) change. Instead of the second relation of (23), in this section we consider a family of Hooke's laws

$$
P^{\lambda}(v)=\frac{1}{\lambda} B \varepsilon(v), \quad \lambda>0,
$$

and perform a passage to the limit as $\lambda \rightarrow 0$. This passage to the limit means that the stiffness of the upper elastic body (upper sheet of the Riemann surface) tends to infinity. Hence, in the limit we can expect an appearance of the upper rigid body (upper rigid sheet). As for the lower body, we obtain a thin inclusion $\gamma$ in it, with suitable boundary conditions on the inclusion faces $\gamma^{ \pm}$.

Now we go back to the general setting of Section 2 (i.e., we do not assume a rectilinear crack geometry accepted in Section 3) and for $\lambda>0$ we consider the functional

$$
\Pi_{\lambda}(u, v)=\frac{1}{2} \int_{\Omega_{\gamma}}\left\{p(u) \varepsilon(u)+\frac{1}{\lambda} P(v) \varepsilon(v)\right\}-\int_{\Omega_{\gamma}}\{g u+h v\},
$$

where $p(u), P(v)$ are defined by the formulae (23).

The minimization problem

$$
\inf _{(u, v) \in K} \Pi_{\lambda}(u, v)
$$

has a solution satisfying the variational inequality

$$
\begin{gathered}
\left(u_{\lambda}, v_{\lambda}\right) \in K, \\
\int_{\Omega_{\gamma}}\left\{p\left(u_{\lambda}\right) \varepsilon\left(\bar{u}-u_{\lambda}\right)+\frac{1}{\lambda} P\left(v_{\lambda}\right) \varepsilon\left(\bar{v}-v_{\lambda}\right)\right\} \\
-\int_{\Omega_{\gamma}}\left\{g\left(\bar{u}-u_{\lambda}\right)+h\left(\bar{v}-v_{\lambda}\right)\right\} \geq 0 \quad \forall(\bar{u}, \bar{v}) \in K .
\end{gathered}
$$


It is clear that the differential formulation of the problem (51), (52) is as follows: find functions $u_{\lambda}=\left(u_{\lambda 1}, u_{\lambda 2}\right), v_{\lambda}=\left(v_{\lambda 1}, v_{\lambda 2}\right)$, such that

$$
\begin{array}{r}
-\operatorname{div} p^{\lambda}\left(u_{\lambda}\right)=g \text { in } \Omega_{\gamma}, \\
-\operatorname{div} P^{\lambda}\left(v_{\lambda}\right)=h \text { in } \Omega_{\gamma}, \\
p^{\lambda}\left(u_{\lambda}\right)=A \varepsilon\left(u_{\lambda}\right), P^{\lambda}\left(v_{\lambda}\right)=\frac{1}{\lambda} B \varepsilon\left(v_{\lambda}\right) \text { in } \Omega_{\gamma}, \\
u_{\lambda}=v_{\lambda}=0 \text { on } \Gamma, \\
u_{\lambda}^{+}=v_{\lambda}^{-}, u_{\lambda}^{-} \nu \leq v_{\lambda}^{+} \nu \text { on } \gamma, \\
p^{\lambda}\left(u_{\lambda}^{+}\right) \nu=P^{\lambda}\left(v_{\lambda}^{-}\right) \nu \text { on } \gamma, \\
p_{\nu}^{\lambda}\left(u_{\lambda}^{-}\right)=P_{\nu}^{\lambda}\left(v_{\lambda}^{+}\right) \leq 0, p_{\tau}^{\lambda}\left(u_{\lambda}^{-}\right)=P_{\tau}^{\lambda}\left(v_{\lambda}^{+}\right)=0 \text { on } \gamma, \\
p_{\nu}^{\lambda}\left(u_{\lambda}^{-}\right)\left(u_{\lambda}^{-} \nu-v_{\lambda}^{+} \nu\right)=0 \text { on } \gamma .
\end{array}
$$

The notations used are similar to those of (21)-(28); for instance,

$$
P_{\nu}^{\lambda}\left(v_{\lambda}\right)=P_{i j}^{\lambda}\left(v_{\lambda}\right) \nu_{j} \nu_{i}, P^{\lambda}\left(v_{\lambda}\right) \nu=P_{\nu}^{\lambda}\left(v_{\lambda}\right) \nu+P_{\tau}^{\lambda}\left(v_{\lambda}\right) .
$$

Substitution of the test functions $(\bar{u}, \bar{v})=(0,0),(\bar{u}, \bar{v})=2\left(u_{\lambda}, v_{\lambda}\right)$ in (52) provides the equality

$$
\int_{\Omega_{\gamma}}\left\{p\left(u_{\lambda}\right) \varepsilon\left(u_{\lambda}\right)+\frac{1}{\lambda} P\left(v_{\lambda}\right) \varepsilon\left(v_{\lambda}\right)\right\}=\int_{\Omega_{\gamma}}\left\{g u_{\lambda}+h v_{\lambda}\right\} .
$$

By the first Korn inequality, from (61) the following estimates are derived:

$$
\left\|u_{\lambda}\right\|_{H_{\Gamma}^{1}\left(\Omega_{\gamma}\right)} \leq c_{1}, \quad \frac{1}{\lambda}\left\|v_{\lambda}\right\|_{H_{\Gamma}^{1}\left(\Omega_{\gamma}\right)}^{2} \leq c_{2},
$$

with constants $c_{1}, c_{2}$ being uniform with respect to $\lambda, 0<\lambda<\lambda_{0}$. Hence, for a subsequence, still denoted by $\left(u_{\lambda}, v_{\lambda}\right)$, we can assume that as $\lambda \rightarrow 0$,

$$
\begin{gathered}
u_{\lambda} \rightarrow u \text { weakly in } H_{\Gamma}^{1}\left(\Omega_{\gamma}\right), \\
v_{\lambda} \rightarrow 0 \text { strongly in } H_{\Gamma}^{1}\left(\Omega_{\gamma}\right) .
\end{gathered}
$$

We introduce the notation

$$
M=\left\{w \in\left(H_{\Gamma}^{1}\left(\Omega_{\gamma}\right)\right)^{2} \mid w^{+}=0, w^{-} \nu \leq 0 \text { on } \gamma\right\}
$$

and substitute in (52) the test function of the form $(\bar{u}, 0), \bar{u} \in M$. Note that $(\bar{u}, 0) \in K$. This implies the inequality

$$
\begin{aligned}
& \int_{\Omega_{\gamma}} p\left(u_{\lambda}\right) \varepsilon(\bar{u})-\int_{\Omega_{\gamma}} g\left(\bar{u}-u_{\lambda}\right)+\int_{\Omega_{\gamma}} h v_{\lambda} \\
& \geq \int_{\Omega_{\gamma}} p\left(u_{\lambda}\right) \varepsilon\left(u_{\lambda}\right)+\frac{1}{\lambda} \int_{\Omega_{\gamma}} P\left(v_{\lambda}\right) \varepsilon\left(v_{\lambda}\right) .
\end{aligned}
$$


Consequently, by passing the lower limit here, we obtain

$$
\begin{gathered}
u \in M, \\
\int_{\Omega_{\gamma}} p(u) \varepsilon(\bar{u}-u)-\int_{\Omega_{\gamma}} g(\bar{u}-u) \geq 0 \quad \forall \bar{u} \in M .
\end{gathered}
$$

The limit problem (62), (63) describes an elastic behavior of the two-dimensional body $\Omega_{\gamma}$ with the thin inclusion $\gamma$. Differential formulation of the problem (62), (63) is as follows: find a function $u=\left(u_{1}, u_{2}\right)$ such that

$$
\begin{array}{r}
-\operatorname{div} p(u)=g \text { in } \Omega_{\gamma}, \\
p(u)=A \varepsilon(u) \text { in } \Omega_{\gamma}, \\
u=0 \text { on } \Gamma, \\
u^{+}=0, u^{-} \nu \leq 0 \text { on } \gamma, \\
p_{\nu}\left(u^{-}\right) \leq 0, p_{\tau}\left(u^{-}\right)=0 \text { on } \gamma, \\
p_{\nu}\left(u^{-}\right) u^{-} \nu=0 \text { on } \gamma .
\end{array}
$$

We omit a derivation of boundary conditions (68), (69), since it can be done in a simpler way as compared to (26)-(28).

Acknowledgement. This work was done during the visit in 2006 of the first author to Keio University, whose support is appreciated very much. It was also supported by the Russian Fund for Basic Research (06-01-00209).

\section{REFERENCES}

[1] Parton V. Z., Morozov E. M. Mechanics of elastoplastic fracture. Moscow, Nauka, 1985.

[2] Morozov N. F. Mathematical questions of a crack theory. Moscow, Nauka, 1984. MR.787610 (87m:73064)

[3] Cherepanov G. P. Mechanics of brittle fracture. McGraw-Hill, 1973.

[4] Khludnev A. M., Kovtunenko V. A. Analysis of cracks in solids. Southampton-Boston, WIT Press, 2000.

[5] Khludnev A. M., Ohtsuka K., Sokolowski J. On derivative of energy functional for elastic bodies with a crack and unilateral conditions. Quarterly Appl. Math., 2002, v. 60, N1, p. 99-109. MR1878261 (2002i:74021)

[6] Kovtunenko V. A. Invariant integrals for nonlinear crack problem with possible contact between crack faces. J. Appl. Mat. Mechs, 2003, v. 67, N 1, pp. 109-123. MR1997626 (2004e:74074)

[7] Kinderlehrer D., Stampacchia G. An introduction to variational inequalities and their applications. New York, London, Toronto, Sydney, San Francisco, 1980. MR.567696 (81g:49013)

[8] Grisvard P. Singularities in boundary value problems. Masson, Springer-Verlag. 1991. MR.1173209 (93h:35004)

[9] Ohtsuka K. Mathematics of brittle fracture. Theoretical studies on fracture mechanics in Japan. Ed. K. Ohtsuka. Hiroshima-Denki Institute of Technology, Hiroshima, 1995. pp. 99-172.

[10] Maz'ya V., Nazarov S., Plamenevskij B. Asymptotic theory of elliptic boundary value problems in singularly perturbed domains, v. 1, 2. Birkhauser, Basel-Boston-Berlin, 2000.

[11] Brokate M., Khludnev A. M. On crack propagation shapes in elastic bodies, ZAMP, 2004, v.55, N2, pp. 318-329. MR2047291 (2005a:74013) 